\title{
Matrimonios mixtos intraeuropeos: un modelo empírico
}

ANTONIO F. ALAMINOS CHICA

IUDESP. UNIVERSIDAD DE ALICANTE

\section{Resumen}

La heterogeneidad con la que nos encontramos al estudiar las parejas interculturales o mixtas, va más allá de las diferencias de origen sociocultural; interviene factores tales como el rol que cada individuo adopta dentro de la pareja (por ejemplo, quién contribuye más económicamente, el status, el nivel educativo, etc.). En este artículo se propone un modelo empírico que muestra el efecto de un conjunto de variables, que expresan circunstancias sociales, sobre la decisión de formar un matrimonio interculturalmente mixto. También las consecuencias en la vida social del individuo.

Palabras clave: matrimonios mixtos, migraciones, aculturación, integración

\section{Abstract}

The intercultural marriages or mixed marriages depend upon several factor. Not only the different cultural origin. Other determinants like the role of the partner (i.e. economic contribution, status, educational level, etc.) or the type of the family (modern, traditional, etc.) influence the outcomes. This paper contains a proposal of empirical model for study the intra-European mixed marriages.

Keywords: mixed marriages, migrations, acculturation, integration, 
Es difícil entender el actual contexto español sin tener en cuenta la multiculturalidad y transculturalidad que deriva del hecho de la creciente concentración y diversidad de la población extranjera en España. Si nos fijamos específicamente en las migraciones europeas, comprobamos que tras la incorporación a la Comunidad Europea las importantes barreras a la movilidad laboral intraeuropea que existían para España desaparecen. A pesar de las predicciones de oleadas masivas de inmigración económica desde los países del sur de Europa hacia países del Europa Occidental y Europa del Norte, sucedió lo contrario: los países europeos del sur y de "cohesión" pasaron, en general, de ser países de emigración neta a ser países de inmigración (Mansoor y Quillin, 2007). Así sucedió con España, convertida recientemente en país de inmigración. A raíz de este cambio, y al aumento también de las migraciones extraeuropeas, la sociedad española cuenta ahora con una mayor presencia de grupos de personas unidos por características étnicas, religiosas, lingüísticas y nacionales propias, dando lugar al hecho social denominado multiculturalidad (Touraine A., 1995).

El fin del siglo $\mathrm{XX}$ ha presenciado un crecimiento sin precedentes en el número de matrimonios mixtos, entre individuos procedentes de diferentes culturas. Especialmente debido a los importantes incrementos experimentados por los movimientos de población (migraciones), consecuencia de diferentes factores. En el caso particular europeo, son escasas las migraciones entre países de la Unión Europea que puedan considerarse forzadas, correspondiendo principalmente con dinámicas de movilidad voluntarias: turismo residencial, formación, movilidad laboral, etcétera. Siendo diferentes las causas que pueden motivar los fenómenos de migración, lo cierto es que la movilidad espacial se esta incrementado y que estas migraciones facilitan las condiciones para la constitución de parejas culturalmente mixtas.

Uno de los modelos sociológicos más influyentes con respecto al establecimiento de matrimonios mixtos con extranjeros procede de las investigaciones de la escuela de Chicago sobre los procesos de asimilación. Desde la perspectiva de la escuela de Chicago, los inmigrantes que llegaban a los Estados Unidos irían progresivamente siendo asimilados hasta adquirir una nueva identidad como norteamericanos. Este modelo teórico enfatiza el carácter gradual y 
múltiple de la asimilación. Un proceso que comienza con la aceptación por parte de los inmigrantes de las costumbres y valores básicos de la sociedad de destino (proceso de aculturación) y termina con la incorporación a la sociedad mediante el matrimonio. En ese sentido, un matrimonio mixto era tomado como indicador del grado de incorporación e integración de los inmigrantes a la sociedad norteamericana.

Ese modelo fue ampliamente criticado, entre otros aspectos, por su presunción de que produciría un movimiento único hacia una cultura común y más o menos homogeneizada. En este proceso los inmigrantes deberían simplemente renunciar a sus pautas y valores de origen para adoptar unos nuevos, producto de sus circunstancias sociales. Esta aproximación ignoraba los diferentes factores que actúan como acelerantes o retardantes, entre ellos por ejemplo, las estructuras de poder y las relaciones de dominación, o la coerción cultural que puede existir dentro de los colectivos de inmigrantes. Por otra parte, no tomaba en consideración en qué grado los inmigrantes adoptarían dichas prácticas y actitudes; en qué contexto y hasta qué punto se incorporan a las relaciones sociales, políticas y económicas de la sociedad de destino.

Los trabajos iniciales sobre matrimonios mixtos desde la perspectiva teórica asimilacionista empleaban como dimensión de control de heterogeneidad la religión. Es decir, las creencias religiosas y especialmente las posibles prescripciones y obligaciones que imponen sobre los estilos de vida y las relaciones cotidianas de los emigrantes. Dentro del campo de visión del análisis se consideraban específicamente y de forma privilegiada tres religiones: el catolicismo, protestantismo y judaísmo. Según esos estudios, los inmigrantes procedentes de diferentes culturas se casaban entre ellos, pero generalmente siempre dentro de los mismos grupos religiosos. Es decir, católicos con católicos, protestantes con protestantes y judíos con judíos. Los matrimonios entre individuos pertenecientes a diferentes grupos religiosos aparecían como una anomalía que debía ser explicada. De acuerdo con ello, la religión de pertenencia supondría el criterio diferenciador máximo y la norma de clasificación de los diferentes tipos de combinaciones interculturales en los matrimonios que consideramos mixtos. Es decir, el aglomerado de referencia, superpuesto y trasversal a las demás diferencias culturales. Por otra 
parte, debe recordarse que estos estudios tomaban como referencia de pareja mixta una definición muy restringida en sentido de normativa legal.

Ciertamente un elemento central cuando se trata de estudiar los matrimonios mixtos es definir la unidad de análisis. A efectos prácticos, consideraremos matrimonios a las parejas que conviven juntas compartiendo un proyecto de vida en común. En ese sentido, el concepto institucional de matrimonio sería solamente una parte del concepto. Así, entre los elementos que concurren entorno a la noción de matrimonio mixto encontramos los aspectos legales, que tienen que ver, por ejemplo, con los derechos y la legitimidad de los hijos. Otro aspecto muy destacado es la posición central que el matrimonio mixto ocupa dentro de la malla social. La familia, como institución social adquiere unas características particulares en aspectos como son la socialización de los hijos, o en la vida doméstica o las rutinas de pareja. Como extensión de esa posición estructural de la familia encontramos, además, los aspectos ceremoniales del matrimonio y de vinculación entre familias. Las posibles diferencias en las estructuras de parentesco, en el sentido de proximidad o visibilidad, deberes y obligaciones hacia la familia. Un famoso dicho advierte que una pareja es el punto de encuentro de dos familias. La noción de familia (como vehículo de tradiciones culturales y religiosas) expresa una unidad de análisis muy importante. Como ya hemos considerado, en los primeros modelos, el aspecto religioso aparece vinculado estrechamente a la noción de matrimonio mixto.

Junto a las dimensiones institucionales y sociales, otro aspecto esencial es la dimensión psicológica o emocional. Complementando claramente las dimensiones sociales, la pareja surge como consecuencia de la relación entre dos individuos. En definitiva, la pareja, es la unidad mínima de convivencia y la más intensa en términos emocionales y de intimidad. Los elementos emocionales que intervienen proceden de educaciones sentimentales, posiblemente diferentes. Tanto sobre el concepto de sexualidad, intimidad, amor romántico, como sus códigos y formas de expresión. En ese sentido, los matrimonios mixtos se refieren a una forma específica de grupo primario. Donde las emociones, los sentimientos y las relaciones de poder son características constitutivas. La forma que adopten estas características dando lugar a tipos de unidades familiares muy diferentes, según el rol 
que adopte cada individuo dentro de la pareja. Por ejemplo, quien contribuye más económicamente, el status, el nivel educativo, etc. Todos ellos son elementos que diversifican los tipos de matrimonios mixtos.

El lenguaje es sin duda una de las expresiones más importantes de la dinámica interna en la pareja mixta. La posibilidad de aprender y emplear dos idiomas, que exista un idioma dominante, y en general las posibles combinaciones que surgen dependiendo del idioma de mayor uso en la sociedad del entorno.

Son, en definitiva, varias las dimensiones que intervienen en ese lugar de encuentro que representa una pareja mixta. Todas ellas podrían, por si mismas, ser la característica o el rasgo diferencial que califica la pareja de mixta o culturalmente híbrida. En los estudios sobre matrimonios o parejas mixtas son diferentes los rasgos empleados para finirla como híbrida. Parejas o matrimonios interculturales significa parejas que proceden de diferentes grupos lingüísticos, religiosos, étnicos o nacionales. En conjunto incluye todas las complejidades de lo que pueda significar una cultura diferente, siendo muy evidente que las diferencias culturales son mayores o menores dependiendo de la procedencia de los componentes de la pareja.

De hecho, llevando al límite el contraste cultural podría hablarse de diferentes subculturas dentro de la misma sociedad. Así algunos autores ${ }^{1}$ destacan que incluso dentro de una misma cultura y clase social existe una división cultural notable debido a otras variables como son el género. Sin embargo, las diferencias entre géneros están presentes en la mayoría de las parejas y no define a una pareja como mixta, por oposición con la mayoría de las parejas en esa sociedad. No puede decirse lo mismo en relación a diferentes clases sociales o incluso regiones dentro de una misma sociedad. En este caso podría decirse que son interculturales de alguna manera específica. La referencia clásica para las diferencias sociales seria sin duda Pigmalion. En definitiva nos habla de las diferentes subculturas asociadas a la clase social, dentro de una misma sociedad. Diferentes normas, usos y costumbres e incluso diferentes escalas de valores. En ese sentido cabe

1 Tannen, D. (1986) That's not what I meant. Nueva York. Ballantine 
destacar la importancia del contexto en el cual se efectúa la definición de mixto o intercultural, el grado de flexibilidad que atribuimos a la definición y el modo en que podrá cambiar.

En definitiva referirse a algo mixto implica la mezcla entre dos cosas diferentes. El criterio que se emplea para definir la diferencia entre ambos será el que de un significado a conceptos como "ego" y "alter". No obstante, no pueden plantearse como absolutas ${ }^{2}$ en la medida que estas diferencias entre grupos culturales vienen definidas por criterios históricos y sin duda cambiantes, como en el ámbito de lo político o de las relaciones socioeconómicas entre ambos grupos.

Las definiciones sobre el concepto de mixto pueden contener una fuerte subjetividad del investigador en la medida que dependa de su punto de vista. En la definición pueden aparecer de forma única o combinada el aspecto económico, social, político o religioso ${ }^{3}$ dentro del proceso para caracterizar la diferencia entre los dos grupos. De acuerdo al criterio adoptado, algunos grupos de emigrantes pueden ser más familiares, su presencia más tolerada o próxima o incluso sus costumbres y prácticas ser admiradas. Es decir, podría llegar a determinar una jerarquía de distancias culturales o de lo que pueda parecer aceptable en lo que se refiere al establecimiento de parejas mixtas ${ }^{4}$. Cruzado sobre lo que pueda ser culturalmente admirable en esta jerarquía de culturas aparece sin duda, los rasgos comunitarios o societarios predominantes en la sociedad. Los términos en que puedan caracterizarse las sociedades o determinados grupos sociales como subculturas societarias o comunitarias, expresan un indicador de proximidad. La solidaridad social basada sobre elementos identitarios mecánicos o que incentivan la imitación (ser igual) implicara sin lugar a duda, una limitación importante para aceptar la diversidad. Por el contrario, las sociedades de lógica más societaria podrían ser mucho más plásticas y flexibles.

Precisamente un ejemplo de ello lo encontramos entre los investigadores sociales. Si los primeros autores tomaban los

2 Barth, F. ed. (1969) Ethnic Groups and Boundaries. The social organisation of culture difference. Bergen. Universitetsforlaget

${ }^{3}$ Dower, J. (1986) War without mercy. London. Faber

${ }^{4}$ Barbara, A. (1989) Marriage across Frontiers. Clevendon 
matrimonios mixtos como indicador de integración de los inmigrantes, otros teóricos percibían los matrimonios mixtos como síntomas de desintegración de la comunidad. Incluso desde la perspectiva de la psicología social, el elegir casarse con una persona de otra cultura podía considerarse como síntoma de conducta desviada. Esa percepción del matrimonio mixto como posible conducta desviada se comprende dentro de un modelo más general, donde solamente las mujeres feas o no deseables podían casarse con gentes de otra cultura. Esencialmente, por qué habían fallado socialmente en el establecimiento de una pareja del mismo status o cultura. Asimismo, se percibía el matrimonio de las mujeres con inmigrantes de mayor estatus como un procedimiento para poder ascender socialmente. Según Merton, los matrimonios mixtos ${ }^{5}$ podrían definirse socialmente por su bajo estatus. Son varios los problemas con este modelo. Además de ser sexista, asume que todas las mujeres desean casarse y que son elegidas, mientras que los hombres eligen. Otros modelos ${ }^{6}$ desarrollan esta presunción del matrimonio mixto como un problema social, centrándose en la experiencia de una mayor fidelidad de las parejas extranjeras. En estas propuestas teóricas, los matrimonios mixtos aparecen como problemáticos ya en la definición misma.

$\mathrm{Y}$ sin embargo, simplemente por el hecho de emigrar a otro lugar no debemos suponer que los individuos son favorables a establecer relaciones sociales en la sociedad de destino. Son múltiples los estudios que muestran la existencia de restricciones culturales en los inmigrantes respecto sus posibles estilos de vida en la sociedad a la que han emigrado. Y viceversa, dado que las restricciones pueden proceder tanto de la competencia y orientación cultural del inmigrante, como de la sociedad de su entorno.

La disposición del inmigrante, y de la sociedad de llegada, a aceptar estilos de vida diferentes es de por si un aspecto central de investigación. Así como, qué factores potencian o atenúan la distancia

5 Merton, R. (1941) "Intermarriage and the social structure" Psychiatry, nº 4, págs. 361-374

6 Imamurra, A. 1990 "Strangers in a strange land: coping with marginality in International Marriage”, Journal of Comparative Family Studies, XXI, n 2, págs 171191. 
social entre grupos culturales. En ese sentido, son varios los factores relevantes.

Por ejemplo, el tiempo que los inmigrantes han vivido en una sociedad está claramente relacionado con la probabilidad de casarse con alguien del país de destino. El tiempo interpretado como experiencia cultural en la nueva sociedad, permite considerar el efecto de las segundas y terceras generaciones de emigrantes. Mientras que la primera generación tiene una probabilidad baja de establecer matrimonios mixtos, ésta se incrementa notablemente para segundas y terceras generaciones. La mayor inserción social de las segundas y terceras generaciones es una variable importante para explicar el mayor nivel de matrimonios mixtos años más tarde de las migraciones originales. Existen, asimismo diferentes estudios que muestran el peso de otras variables como son el género del inmigrante. Es significativamente mayor el porcentaje de mujeres que se casa con extranjeros. Porcentaje sensiblemente mayor que el de hombres que se emparejan con extranjeras. En este caso, juega su efecto tanto la magnitud de las cifras, como el control cultural sobre la mujer. Primero, la mayor importancia de la migración masculina. Determinados flujos migratorios son eminentemente masculinos, incrementándose la probabilidad de emparejar con una mujer nativa. En segundo lugar, de existir, es posible un mayor control cultural en el grupo inmigrante con respecto a la mujer. Con ello, las probabilidades de encontrar pareja en la sociedad de llegada es menor para las mujeres sobre las que pueda existir un mayor control familiar. Un ejemplo frecuente es el caso de las mujeres argelinas en Francia, que tienen restringido su contacto con los franceses. En ese sentido, el mayor porcentaje de mujeres occidentales que se casa con extranjeros podría explicarse de las condiciones especiales de la migración, con una menor movilidad femenina y restricciones familiares en la cultura de origen de la mujer. En el caso que nos ocupa, la mayor proporción de mujeres que se casa con europeos de otro país encontraría una explicación más directa en el mayor volumen de la migración masculina intraeuropea que en restricciones culturales hacia la mujer. Los datos estadísticos revelan que son mucho más las mujeres que se casan con extranjeros en Estados Unidos, Alemania o Francia. Aproximadamente un 60\% son mujeres. Probablemente las cifras, o en todo caso la tendencia sea similar para el resto de los países europeos. También juega un papel importante que con el incremento de los derechos de las mujeres, 
especialmente en Europa, éstas tienen una mayor libertad para establecer parejas y matrimonios con extranjeros. Sin embargo no podemos olvidar que aunque existe cada vez más esta libertad, no por ello dejan de asistir barreras informales, tales como discurso negativo sobre los extranjeros o estereotipos que pueden influir en cómo reacciona el entorno familiar o próximo de la mujer para establecer una pareja con una persona de otra cultura ${ }^{7}$.

Otro asunto interesante en el sentido de establecer contextos poco apropiados para las parejas mixtas, es la propuesta de Cohen acerca de la "organización formal de la etnicidad", donde se genera de una forma consciente, una forma específica de organizar la política y la economía entre los inmigrantes. Tanto en el ámbito del trabajo como en los servicios surgen espacios segregados que se convierte en enclaves étnicos. Así, se forman pequeños mercados protegidos en el que desarrollar una actividad identitaria excluyente actúa en beneficio de los empresarios étnicos. Potenciar los sentimientos de solidaridad dentro del grupo de inmigrantes es parte del mercado étnico. De esta forma, intentan crear un mercado cautivo. Los empresarios tienen una doble interés, económico y emocional, en que se mantenga la creencia y participación en la comunidad de inmigrantes. La generación de mercados y espacios étnicos actúan como frenos al establecimiento de parejas mixtas.

Por otra parte, muchas de las antiguas barreras al matrimonio mixto están desapareciendo. Por ejemplo el descenso de la importancia de la religión a la hora de regular como se vive la vida cotidiana y el incremento en la proceso de secularización tanto en Europa como en Norteamérica. En los modelos de secularización, la similitud educacional y profesional ha se considera como factor que posibilita la formación de matrimonios interculturales. En el caso de matrimonios intraeuropeos, destaca la migración que podría considerarse cualificada, en la que los dos individuos que forman una pareja mixta tienen un nivel educativo importante. En las migraciones actuales la calificación profesional y la educación es un capital fácilmente transportable y que

7 Breger, R. (1992) "The discourse on Japan in the German Press: Images of Economic Competition”, en R. Goodman y K Refsing (eds.) Ideology and Practice in Modern Japan. London. Routledge

${ }^{8}$ Cohen, A. (1974) Urban Ethnicity. ASA, 12, London. Tavistock. 
permite mayores oportunidades a los individuos que emigran, tanto de trabajo como de interacción social. Este planteamiento contradice los modelos iniciales sobre el bajo estatus de los matrimonios mixtos. Sin embargo, en la actualidad son varios los estudios descriptivos indicando que los matrimonios mixtos lo forman predominantemente las personas de bajo y medio status, con poca educación. Sin duda, en el debate sobre la importancia de la religión, la clase social o la educación en el momento de elegir pareja es necesario cualificar que grupos socioeconómicos, y culturales están definiendo el carácter de mixto. Los matrimonios mixtos de españoles con noruegos, ecuatorianos, magrebíes, franceses, ecuatorianos, etc. mostraran perfiles muy diferentes. Así, un aspecto muy importante son los indicadores de percepción de distancia social en el momento de influir la decisión sobre los grupos que pueden proveer de parejas potenciales y que grupos simplemente no se consideran. Es decir, la existencia no explicita socialmente, de una jerarquía de aceptabilidad, en lo que se refiere a la cultura de origen de la pareja. En paralelo con los escalamientos de racismo donde uno de los ítems de referencia es la disposición a que "su hija se casase con "un grupo étnico diferente (asiáticos, afro americanos, etc.).

Waldren señala ${ }^{9}$ como las líneas definitorias entre los grupos que pueden definir las parejas mixtas han cambiado durante el siglo XIX y XX, afectando con ello a la elección y aceptación del las parejas. El cambio en las relaciones sociales y económicas, así como el incremento del contacto con extranjeros después de la segunda guerra mundial, fue acompañado de una fascinación con la diferencia. En su opinión, la combinación de factores anteriores incrementó el número de los matrimonios mixtos que podían considerarse exóticos, con parejas de no españoles, en ocasiones procedentes de diferentes clases sociales o religiones.

En un matrimonio mixto pueden existir diferentes grados de percepción de las diferencias. Mientras que algunas pueden ser positivas, otras pueden suponer puntos de conflicto. Esta percepción y evaluación de las diferencias no procede un exclusivamente de la

9 Waldren J. (1998) Crossing over: Mixing, matching and marriage. En Bregar, R y Hill, R. Cross-cultural marriage. Identity and Choice. Oxford. Berg. 
familia más próxima, sino también de la familia extensa o la comunidad en la que se vive. La familia puede salir fortalecida si existe algo más importante que supere la percepción de las diferencias. Yamani documenta ${ }^{10}$ como el trasladarse a una nueva localidad y con ello escapando de la presión social del entorno, puede ayudar a mantener el matrimonio. Un entorno más neutral o, en general, el lugar donde vive una pareja mixta, parece ser esencial para permitir una cierta flexibilidad en la negociación que establezca la pareja con respecto a sus expectativas culturales diferentes. En ese sentido, es importante considerar la presión social del entorno como contexto de las negociaciones domésticas que establezca la pareja.

En otras ocasiones los que se implican en un matrimonio mixto pueden en un momento inicial no ser conscientes de lo diferentes que pueden llegar a ser sus creencias y prácticas, lo que puede producir un desajuste en las expectativas y un creciente sentimiento de frustración. Así las diferencias en los rituales cotidianos, como son la atención, conversaciones, el empleo del espacio y la privacidad, así como la comida están fuertemente vinculados a las estructuras familiares de poder y jerarquías. La expectativas asociadas a los diferentes roles y concepciones de la privacidad son en ocasiones claramente divergentes. Con frecuencia los conflictos entre las expectativas y la experiencia son posteriores al establecimiento de la pareja mixta, y surgen como consecuencia de las relaciones cotidianas.

También debemos considerar que junto con los rasos diferenciales se encuentran otros que producen semejanzas entre ellos $^{11}$. Dos personas procedentes de diferentes culturas pueden tener más en común que diferencias, especialmente si comparten una serie de características como pueden ser estilos de vida urbana, educación elevada, una misma experiencia profesional, etc. Es decir, aquello que define la diferencia cultural en los matrimonios mixtos puede verse traspasado por otras dimensiones que suavizan o intensifican dichas diferencias. Por ejemplo si definimos como mixto el matrimonio entre una persona española y otra francesa, las diferencias pueden

${ }^{10}$ Yamani M.(1998) “Cross-Cultural Marriage within Islam: Ideals and Reality” en Breger y Hill Cross-cultural Marriage. Identity and Choice”.Oxford. Berg.

11 Cottrell, A. (1990) "Cross-national marriages: A review of the literature". Journal of Comparative Family Studies. XXI. n ${ }^{\circ}$ 2. pag 151-169. 
intensificarse o suavizarse en función de las combinaciones de otras características como puedan ser clase social de origen, profesión, nivel educativo, religión etc.

En un nivel íntimo y personal, entra en consideración con mayor fuerza la noción de personalidad. Conjuntamente con los estereotipos sociales, encontramos en el ámbito de lo individual una mayor o menor disponibilidad o deseo para aceptar las diferencias de la pareja. En definitiva, desde una perspectiva micro, se reconstruyen los conceptos de integración social, desde su formulación como interacción social. Mientras que el concepto de integración social considerado desde una aproximación macro produce una cierta simplificación, necesaria para poder efectuar generalizaciones, el enfoque micro reconoce el peso especifico de las diferencias individuales y en ese modo, describe y percibe una mayor variabilidad.

Desde lo macro, el método parte de estereotipar e intentar generar arquetipos y simplificaciones de las prácticas culturales. Eso tanto para quien vive en esa cultura, como para el que es extranjero y extraño a ella. La labor de las simplificaciones son especialmente efectivas y evidentes en ocasiones especiales ${ }^{12}$ como son los conflictos, guerras, campañas políticas y en general los momentos de movilización nacional. Cuando en lugar de sociedades en conjunto es el caso de las parejas mixtas, se destaca casi necesariamente la posibilidad de que las convenciones culturales sean diferentes ${ }^{13}$. Es fácil encontrar una relación de actividades y valores donde se detalla como las personas de una cultura lo hacen de una forma, y los de otra cultura actúan de forma diferente. De este modo ciertamente el diseño de la investigación presupone que existe una cultura homogeneizada y que de este modo las convenciones culturales, incluyendo religión, lengua, normas, usos, y expectativas son en general las mismas para las personas que forman parte de un mismo grupo étnico o cultura. Ciertamente los estereotipos, y con ellos las generalizaciones, simplifican e ignoran la diversidad social. La atribución de

12 Bachu, P. (1993) "Identities constructed and reconstructed: Representation of Asian women in Britain", en G. Buijs (ed.) Migrant Women. Crossing Boundaries and Changing Identities. Oxford. Berg.

13 Johnson, W. and Warren M. (eds) (1994) Inside the Mixed Marriage. Lanham: University Press of America. 
características a un grupo, incluido el carácter nacional, se supone natural inherente y en cierto modo resistente al cambio. Ese carácter naciona $1^{14}$ es parte de la diferencia entre ellos y nosotros. Sin embargo esas fronteras no son simplemente una descripción pasiva sino que por el contrario incorporan juicios de valor ${ }^{15}$ con respecto a esa diferencia potencial. El establecimiento de diferencias y la propuesta de estereotipos están cargados emocionalmente. Por otra parte, esto anima a que la gente a buscar esposa o pareja dentro de su propio círculo. Por otra parte, el carácter positivo negativo de los estereotipos con respecto a la pareja extranjera influye sin ninguna duda en la valoración social. Algunos autores destacan como los estereotipos negativos existentes entre los grupos puede actuar como filtro en el momento de seleccionar ${ }^{16}$ una posible pareja.

En ese sentido, la simplificación y homogenización que se presupone al considerar un grupo étnico o nacional cualquiera ha sido objeto de una crítica frecuente. Para algunos, los antropólogos sobrestiman y conceden una importancia excesiva a la homogeneidad cultural, producto de que su objeto de estudio considera tratar con tribus o grupos sociales muy pequeños, ciudades o grupos étnicos en áreas urbanas.

Sin embargo, debemos hacer hincapié, especialmente en el contexto de los matrimonios mixtos, en qué la homogeneidad intragrupo (etnia, nación, etc.) es el resultado de un conflicto ${ }^{17}$ ideológico. Incluso en el caso teórico que dentro de cada grupo no existiese ninguna variación sobre la pauta que se propone como general. Los rasgos más generales son el resultado de un conflicto con respecto a cual deba de ser la adecuada. Por ello, lo más realista es asumir que existe un amplió abanico de usos y costumbres, así como normas dentro de un grupo étnico, que puedan constituir un núcleo

14 Said, E. (1978) Orientalism. London. Routledge. Steadman, P. (1969) The Myth of Asia. New York. MacMillan. Dower, J. (1986) War Without Mercy. Race and Power in the Pacific War. London. Faber

${ }^{15}$ Foucault, M. (1974) La arqueología del saber.

${ }^{16}$ Spickard, P. (1989). Mixed Blood. Intermarriage and Ethnic identity in Twentieth-Century America. Madison. University of Wisconsin Press

17 Hobsbawm, E.; Ranger, T (eds.) (1983) The invention of tradition. Cambridge. Cambridge University Press. 
central más generalizado de creencias o de prácticas, siendo otras mucho más flexible y variables ${ }^{18}$, constituyendo una especie de periferia donde se tolera en un mayor grado las diferencias. Las costumbres o prácticas culturales que se pueden considerar generalizadas experimentan cambios, tanto desde el proceso de interacción entre individuos, como con las modificaciones en los entornos sociopolíticos y económicos que nos rodea. Sin embargo, debemos considerarlas en la vigencia actual y su actuación sobre los miembros de la pareja. Los matrimonios mixtos, los individuos que forman la pareja, están sujetos por tanto a la doble sanción de este tipo de normas o valores, correspondientes a cada intra-grupo. De este punto de encuentro que suponen los matrimonios mixtos pueden surgir resultados muy diferentes en lo que se refiere a la combinación e hibridación de normas y estilos de vida o, por otro lado, a la imposición hegemónica de la cultura de uno de los miembros de la pareja.

En todo caso, la apreciación por parte de los componentes de la pareja de las diferencias dentro de una pareja mixta, dependen en gran parte del lugar que ocupa la pareja en el ciclo vital así, como de su situación socioeconómica. Así, la percepción de las diferencias poco a poco deja de ser culturales para convertirse en personales. Es decir, que las diferencias dentro de la pareja mixta tienden a concentrarse en torno a las diferencias entre las personalidades de cada individuo y no tanto a presiones procedentes de la variación cultural. En el caso extremo, de excelente afinidad entre las personalidades, puede llegar a negarse la diferencia cultural, especialmente en parejas con perfiles profesionales y educativos muy semejantes. De esta forma, se reducen las diferencias que se perciben en la pareja y se atribuyen a la cultura en general, cuyo peso se relativiza. De hecho, la diferencia puede llegar a ser parte del atractivo que una persona encuentra en su pareja, dentro de un matrimonio mixto. Es decir, la diferencia puede ser atractiva.

Ciertamente son varios los factores sociales que influyen en el momento de elegir una pareja extranjera. Los diferentes estereotipos y prejuicios que puedan existir tanto en los individuos como en el

18 MacDonald, M. (1994) "Women and Linguistic Innovation in Britany", en P. Burton, K.K. Dyson y S. Ardener (eds.) Bilingual Women, Anthropological Approaches to Second Language Use. Oxford. Berg. 
entorno social en el que vive. Sin embargo otro elemento esencial es sin lugar a duda la elección personal. Según algunas investigaciones, existen diferencias de género en relación a la elección de una pareja extranjera. Mientras que las mujeres parecen preocuparse menos por ser extranjeros y concentrarse más en la sensación de haber encontrado a la persona correcta. Pocas mujeres se preocupan de indagar en profundidad sobre la cultura de su futura pareja, de su familia o país de origen antes del matrimonio, y no parece importarles demasiado. Esta conclusión aparece en diferentes estudios que contemplan de forma retrospectiva la elección, pensando en el momento en que se produjo la decisión de tener una pareja extranjera.

En todo caso, debemos considerar que el establecimiento de una pareja mixta en términos legales, como pueda ser el matrimonio, requiere la intervención de un tercer actor: el Estado o la administración pública. En las sociedades occidentales desarrolladas, se asume con frecuencia que las limitaciones que puedan afectar a la elección de una pareja o el éxito que tenga el matrimonio, es esencialmente de carácter informal y relacionado con la convivencia. Sin embargo, un conjunto importante de limitaciones juegan un papel significante, tanto en el sentido de situación legal del inmigrante, como en la formalización de matrimonios como parte del control de los derechos de ciudadanía de los no nacionales.

\section{Un modelo empírico}

Como hemos podido apreciar, son varias las dimensiones implicadas en el momento de intentar dar una explicación al incremento de matrimonios entre personas de diferente cultura o nacionalidad. Tomadas todas ellas en conjunto, puede configurarse una explicación que permita una argumentación lógica del fenómeno social y sus antecedentes contrastable con la información disponible. Es decir, en lugar de comprobar la validez empírica de cada una de las hipótesis (que forman parte de la explicación) vamos mediante el método de análisis de estructuras de covarianzas, evaluar y diagnosticar como se apoya, en que grado y con que significación, toda la argumentación que empleamos se sostiene en base a las relaciones empíricas conocidas.

La explicación que vamos a testar empíricamente empleará datos de una población muy heterogénea. Es decir, considerará su 
posible ajuste sobre una muestra formada por españoles, alemanes, franceses, italianos y británicos emigrados a los otros países. Por ejemplo, alemanes en Francia, España, Italia y Gran Bretaña. La heterogeneidad no procede tanto de las diferentes nacionalidades de origen como de las diferentes condiciones para la migración y con ello los perfiles sociodemográficos que contienen. Así, los alemanes en Francia, España o Italia están más envejecidos como media y muchos de ellos jubilados, mientras que los españoles o italianos en Francia o Alemania son más jóvenes y buscando mejorar las condiciones laborales y de forma de vivir. Es en ese sentido que consideramos heterogénea la población sobre la que se testara empíricamente el modelo. Ciertamente, ajustarlo independientemente para cada subpoblación mejoraría sensiblemente todos los indicadores, al responder a grupos más homogéneos en sus condiciones sociales y estilos de vida. Ajustar sobre la subpoblación presenta sin duda beneficios empíricos. Sin embargo, consideramos de mayor interés el beneficio teórico que representa las explicaciones trasversales a las diferentes circunstancias sociales. En ese sentido, el modelo teórico propuesto para su comprobación empírica lo va a ser en condiciones de una población muy heterogénea y en ese sentido, adversas a un modelo general. En otro sentido, el modelo explicativo será especialmente significativo en lo que se refiere al peso de las variables involucradas para comprender las circunstancias que activan el fenómeno de los matrimonios mixtos.

Las dimensiones y variables contempladas teóricamente como relevantes para especificar el modelo explicativo han sido las ya consideradas previamente en las páginas iniciales al revisar los antecedentes teóricos.

Considerando el matrimonio o el establecimiento de parejas con individuos de otras culturas, es necesario pensar en qué condiciones es factible que los individuos se encuentren, las oportunidades que existen para qué una pareja llegue a conocerse. Así, las condiciones que facilitan la formación de parejas mixtas son el objeto central de análisis empírico de este artículo. No obstante, no es posible eludir dos consecuencias psico-sociales del establecimiento de parejas mixtas: como son sus consecuencias en el sentido de sentirse más discriminados por la sociedad del entorno, así como un refuerzo de las redes sociales de pertenencia. Ciertamente, no son aspectos 
contradictorios Las probabilidades de sentirse discriminados se incrementan cuando se incrementan las posibilidades de contacto social. La mayor participación y actividad social incrementa las circunstancias y oportunidades para sentirse tratado de forma desigual respecto a los demás. Por ello, un elemento central, como antecedente y consecuente es el grado de integración social, en términos de conocimiento y participación en redes informales de conocidos y amigos, o en el contexto laboral. Como variables explicativas exógenas, se han considerado las mediciones sobre "competencia lingüística" (indicador producto de la combinación de tres variables), el género del emigrante, la edad en el momento de llegar al país donde reside actualmente y la situación laboral. Ciertamente las circunstancias de aquellos que llegan en condiciones de incorporarse al mercado de trabajo son en general bastante diferentes a los que emigran por otras razones.

La estructura relacional se muestra en términos teóricos, mediante un sistema de hipótesis expresado matricialmente. La matriz de efectos nos muestra, empleando el coeficiente unidad, allí donde postulamos la existencia de una relación explicativa. El signo expresa la forma que proponemos para dicha relación. En conjunto, la forma matricial sintetiza la propuesta explicativa, de modo que el proceso argumental es apreciable de forma directa y simultanea.

Posteriormente, se presenta como diagrama el ajuste empírico, incorporando los coeficientes producto del análisis. Coeficientes estandarizados que nos hablan tanto de la intensidad relacional entre las proposiciones de la hipótesis condicional, como del ajuste en conjunto de la argumentación.

Así, la probabilidad de establecer un matrimonio mixto es mayor para los hombres emigrantes, que trabajan al llegar a la sociedad de destino (se incrementa el contacto social), jóvenes y que no muestran añoranza o deseos de regresar. La estrategia vital adoptada es fundamental, en la medida que los emigrantes pueden orientarse a empezar una nueva vida en el país de destino, o basarse en la esperanza del regreso. Lógicamente, la probabilidad de establecer pareja con alguien con la nacionalidad de la sociedad donde se emigró se incrementa cuando el individuo se propone una nueva vida. Por su parte, el establecer un matrimonio mixto (pareja mixta) incrementa la red social del individuo emigrado (a través de la red de la pareja) y, 
simultáneamente, la percepción de haber sido discriminado. Esta sensación de discriminación es mayor en las mujeres que en los hombres, y especialmente entre aquellos que mantienen la esperanza de regresar, y que se sienten más insatisfechos con su vida. Por el contrario, la satisfacción con la vida en la sociedad de destino es mayor entre los que se integran más intensamente (red social) y decidieron construir su vida en la nueva sociedad.

Podemos apreciar como, desde la perspectiva de un modelo global, se validan varias hipótesis tradicionales, como son el mayor porcentaje de hombres emigrantes, la vinculación entre establecer parejas interculturales y una mayor integración social, así como la satisfacción con la vida en la sociedad de residencia. Por último destaca la importancia de la competencia lingüística. Un conocimiento adecuado del idioma de la sociedad a la que se emigra facilita la integración en redes sociales, y se asocia estrechamente a la estrategia vital de comenzar una nueva vida. Una baja competencia lingüística incrementa la probabilidad de desear el regreso y poseer una red social menos densa.

Tabla 1: Matriz de efectos modelo general: Migración y Matrimonios mixtos (EIMMS )

\begin{tabular}{|c|c|c|c|c|c|c|c|c|c|c|}
\hline & 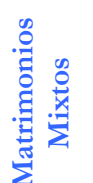 & 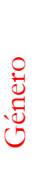 & 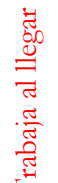 & 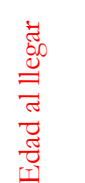 & 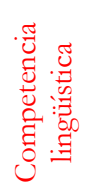 & 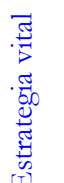 & 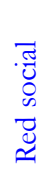 & 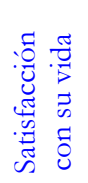 & 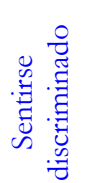 & 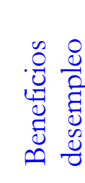 \\
\hline $\begin{array}{l}\text { Matrimoni } \\
\text { o Mixto }\end{array}$ & & 1 & 1 & -1 & & 1 & & & & \\
\hline $\begin{array}{l}\text { Estrategia } \\
\text { vital }\end{array}$ & & & & 1 & 1 & & & & & \\
\hline Red social & 1 & & 1 & & 1 & 1 & & & & \\
\hline $\begin{array}{l}\text { Satisfacción } \\
\text { vida }\end{array}$ & & & $\begin{array}{l}-1 \\
\text { (a) }\end{array}$ & & & 1 & 1 & & -1 & \\
\hline $\begin{array}{l}\text { Discrimina- } \\
\text { do }\end{array}$ & 1 & -1 & 1 & -1 & & $\begin{array}{l}-1 \\
\text { (b) }\end{array}$ & & & & 1 \\
\hline $\begin{array}{l}\text { Benef. } \\
\text { Desempleo }\end{array}$ & & & 1 & $-1(\mathrm{c})$ & & & & & & \\
\hline
\end{tabular}


(a) $\mathrm{Al}$ ser un modelo global, los jubilados que emigran por calidad de vida son más felices (alemanes y británicos en España) que los que aún son población activa.

(b) Los que adoptan una estrategia de retorno se sienten más discriminados. Orientados a su país.

(c) Los que inmigraron más jóvenes, ahora tienen mayor probabilidad de haber pedido ayudas de desempleo.

Controlado por la edad actual, los de mayor edad son los que con mayor probabilidad han pedido ayudas de desempleo.

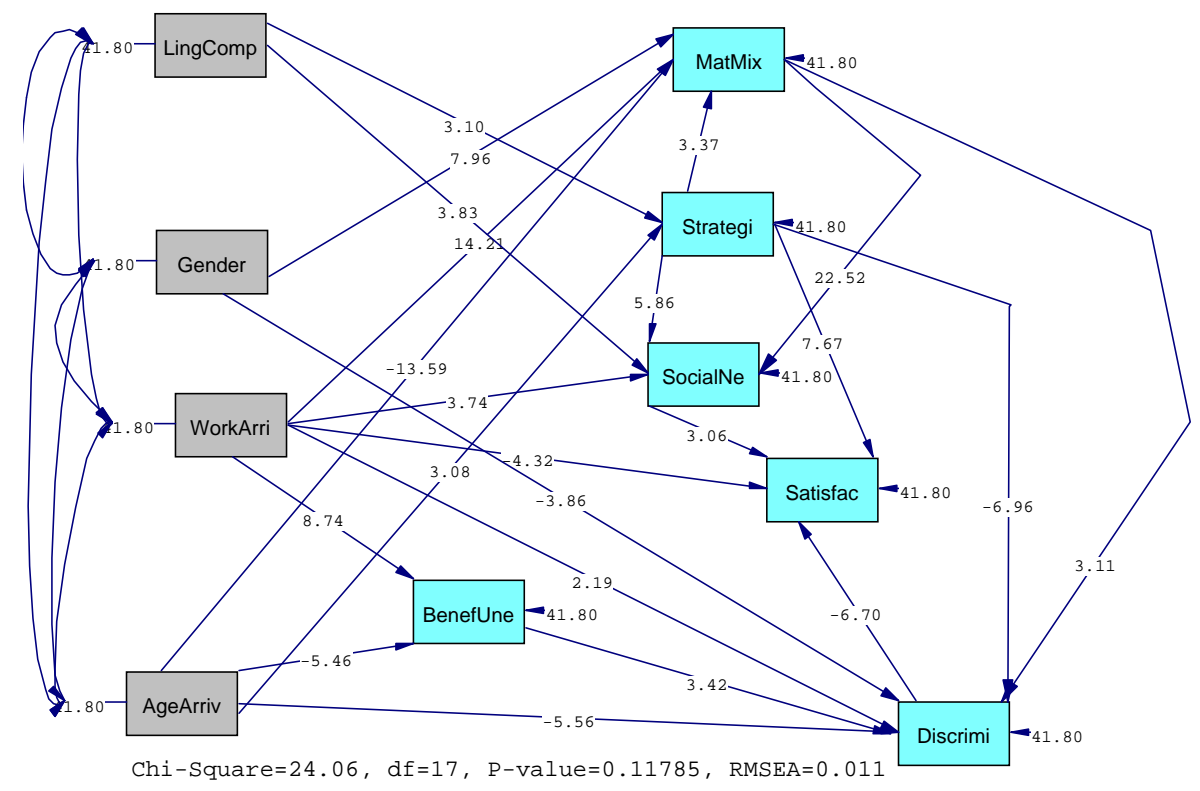

